\title{
Alternative Pathway of Complement Activation in Full Term and Premature Infants
}

\author{
ROBERT C. STRUNK, ${ }^{(18)}$ LAWRENCE J. FENTON, AND JOHN A. GAINES \\ From the Department of Pediatrics and the Division of Computer Systems and Biostatistics, Arizona Health Sciences \\ Center, University of Arizona, Tucson, Arizona, USA
}

\section{Summary}

Classical and alternative pathway complement levels were measured in the cord blood sera of 60 newly born infants, with weights ranging from 1200-4165 g. The impact of maternal illness and infant illness on the complement levels was also evaluated. The mean values for $\mathrm{CH50}, \mathrm{C3}, \mathrm{C4}$, $\mathrm{PH} 50$, factor $\mathrm{B}$, and properdin were all significantly less than normal adult levels $(P<0.0001)$. All of the above determinations were significantly correlated with one another except for the relationship between properdin and factor $B$.

$\mathrm{CH} 50, \mathrm{PH} 50, \mathrm{C4}$, and properdin levels were significantly correlated with birth weight although there was much residual scatter. Neither maternal illness nor mild to moderate illness in the newborn altered the birth weight-complement relationships. Severe infant illness did significantly alter the relationship between birth weight and complement. However, the impact of this variable on the birth weight-complement relationships was not consistent among the various components. These inconsistencies and the small sample size preclude drawing any strong conclusions about severe illness and complement levels.

\section{Speculation}

The alternative pathway activation sequence appears to be deficient to the same degree as the classical pathway activation sequence. This suggests that the entire serum complement system develops as a single unit. Severe illness in very low birth weight babies may be associated with abnormalities in serum complement levels greater than would be expected from the low birth weight alone.

The high mortality and morbidity from sepsis and meningitis in the newborn is closely related to the lack of development of the newborn's humoral and cellular defense mechanisms (14). The serum complement system is one of the major humoral defense mechanisms required for effective clearance of bacteria (7). Several investigators have found both the activity of the classical complement pathway and levels of its specific components are low in infant cord blood when compared to adult levels $(1,3,6,13)$. The degree of depression of these levels tends to correlate positively with birth weight. Stossel et al. (15) have suggested that low levels of alternative pathway components may be more important in predisposing infants to infection than defects of the classical pathway.

The purpose of this study is to evaluate levels of properdin and factor B of the alternative complement pathway, as well as the function of the alternative complement pathway, in full term and premature infants. In addition, the impact of maternal and infant illness on both classical and alternative complement levels is analyzed.

\section{MATERIALS AND METHODS}

\section{PATIENTS}

Sixty infants born at Arizona Health Sciences Center from January to June, 1975 were studied.

Cord blood was collected by venipuncture from the placental vessels within $10 \mathrm{~min}$ of delivery of the placenta, allowed to clot in sterile glass tubes, and refrigerated at $4^{\circ} \mathrm{C}$ for no more than 16 $\mathrm{hr}$. Serum was then obtained after centrifugation at $0^{\circ} \mathrm{C}$, aliquoted, and frozen at $-70^{\circ} \mathrm{C}$ until assayed for complement. The gestational age of all infants was determined by using maternal dates and Dubowitz Scoring System on admission to the nursery. Histories from newborn and maternal charts were recorded on standard computerized history summary sheets used by the Neonatology section for all deliveries at Arizona Health Sciences Center.

The weights of the infants ranged from $1200-4165 \mathrm{~g}$. Eleven infants were small for gestational age. A total of 18 newborns were classified as having illness which was considered "mild": 14 with fetal distress (but good Apgars); three with idiopathic respiratory distress syndrome not requiring ventilatory support, and one with transient hypotension at birth. Six infants, all under $1500 \mathrm{~g}$, evidenced illness considered "severe": five idiopathic respiratory distress syndrome (all required ventilatory support; one developed a coagulopathy and died), and one birth asphyxia (Apgar $<3$ at $1 \mathrm{~min}$, and $<5$ at $5 \mathrm{~min}$ ). None of the infants showed signs of any infection. Twenty mothers were considered "ill" for the purposes of the study. The illnesses were: preeclampsia (9), diabetes (3, class B or greater), urinary tract infection at delivery (2), amnionitis (1), partial abruption (1), methadone addiction (1), idiopathic thrombocytopenic purpura (1), porphyria (1) and Hodgkins disease (1). Three of the infants of these 20 sick mothers had severe illness, seven had mild illness, and four were small for gestational age.

\section{COMPLEMENT STUDIES}

The third (C3) and fourth (C4) components of complement and factor B and properdin levels were determined by radial immunodiffusion using the method of Mancini et al. (8). Antisera were obtained from Atlantic Antibody, Westbury, Maine. Reference serum from Atlantic Antibody was used to compute the standard curve in each assay of each specific protein, except for properdin where a single normal adult serum was used as $100 \%$. Whole complement activity, $\mathrm{CH} 50$, was measured by standard methods (9).

Functional activity of the alternative complement pathway (properdin hemolysis $50 \%$ or PH50) was determined using a modification of the method described by Platts-Mills and Ishizaka (10). Whole blood was obtained from adult New Zealand albino rabbits and stored as a 50\% solution with sterile Alsever's solution. Before use the rabbit red blood cells ( $\mathrm{RaRBC}$ ) were washed four 
times with veronal buffered saline containing $0.01 \mathrm{M}$ EDTA (EDTA buffer) (12), and three times with veronal buffered saline containing $0.08 \mathrm{M}$ ethylene glycoltetra-acetic acid (EGTA) and $0.008 \mathrm{M} \mathrm{Mg}^{++}$(Mg-EGTA buffer). One hundred $\mu$ l RaRBC, $1 \times$ $10^{8}$ cells $/ \mathrm{ml}$ in Mg-EGTA buffer, were incubated at $37^{\circ} \mathrm{C}$ with equal volumes of Mg-EGTA buffer and one of six serum dilutions in Mg-EGTA buffer (from 1/10 to 1/33). After $2 \mathrm{hr}$ of incubation, $3 \mathrm{ml}$ of EDTA buffer were added to each tube and the remaining RBC sedimented. After the supernatants had been discarded, the cell buttons were lysed with $1.5 \mathrm{ml} \mathrm{H}_{2} \mathrm{O}$ and the optical density at $412 \mathrm{~nm}$ determined. The serum dilution yielding 50\% hemolysis was calculated (12).

Complement values in sera taken from a random sample of 33 normal adults were measured in the same way to provide mean values for comparison with the neonatal levels.

\section{STATISTICAL ANALYSIS}

Each complement measurement was analyzed with respect to birth weight. Differences in this relationship attributable to the four infant variables (well, mild illness, severe illness, and small for gestational age) and two maternal variables (well and ill), all entered as dummy variables, were evaluated using saturaged model analyses of covariance of the dependence of complement on birth weight in a stepwise multiple regression program.

\section{RESULTS}

The only variable which significantly altered the basic relationship between birth weight and complement was "severe infant illness". The impact of this variable on the birth weight-complement relationships was not consistent among the various components. Because of these inconsistencies and the small sample size, this finding was not considered any further, and the analysis was repeated excluding these six cases of "severe infant illness". That analysis showed that the remaining variables did not significantly alter the relationship between complement components and birth weight. Thus, a single regression line for each complement component on weight is an adequate summary of the basic relationship between the two variables.

The results of the complement studies for the 54 infants remaining after the 6 infants with severe illness were excluded are shown in Table 1 . The mean values were significantly below the normal adult levels $(P<0.0001)$ for each component. None of the variables were notably nonnormal in distribution.

Scatter plots of the classical and alternative complement path-

Table 1. Complement levels in infants and adults

\begin{tabular}{lcccc}
\hline \multicolumn{1}{c}{ Variable } & \multicolumn{2}{c}{ Infants } & & \\
& $n$ & Mean & SD & Range \\
\hline Weight & 54 & 2695 & 823.7 & $1200-4165$ \\
CH50, functional units & 36 & 23.3 & 8.7 & $0-38$ \\
PH50, functional units & 54 & 13.6 & 3.8 & $6-22.8$ \\
C3 (mg/dl) & 54 & 71.5 & 17.9 & $33-109$ \\
C4 (mg/dl) & 44 & 17.2 & 7.1 & $5.3-32.5$ \\
B (mg/dl) & 53 & 14.2 & 5.6 & $7.1-35.2$ \\
Properdin (\% normal adult & 53 & 71.8 & 21.3 & $35-125$ \\
$\quad$ serum) & & & &
\end{tabular}

Normal Adults

CH50, functional units

PH50, functional units

$\mathrm{C} 3(\mathrm{mg} / \mathrm{dl})$

$\mathrm{C} 4(\mathrm{mg} / \mathrm{dl})$

B (mg/dl)

Properdin $(\%$ normal

adult serum)

\footnotetext{
' See text for details.
}

Table 2. Bivariate regressions with weight

\begin{tabular}{lccc}
\hline Dependent variable & Intercept & Slope & $r$ \\
\hline CH50 & 8.02 & 0.0056 & $0.49^{1}$ \\
PH50 & 8.47 & 0.0019 & $0.41^{2}$ \\
C3 & & & $0.21^{3}$ \\
C4 & 10.20 & 0.0028 & $0.32^{2}$ \\
B & & & $0.13^{3}$ \\
Properdin & 55.39 & 0.0060 & $0.23^{2}$ \\
\hline
\end{tabular}

' $r$ significant beyond 0.01 level.

${ }^{2} r$ significant beyond 0.05 level.

${ }^{3}$ Regression not significant.
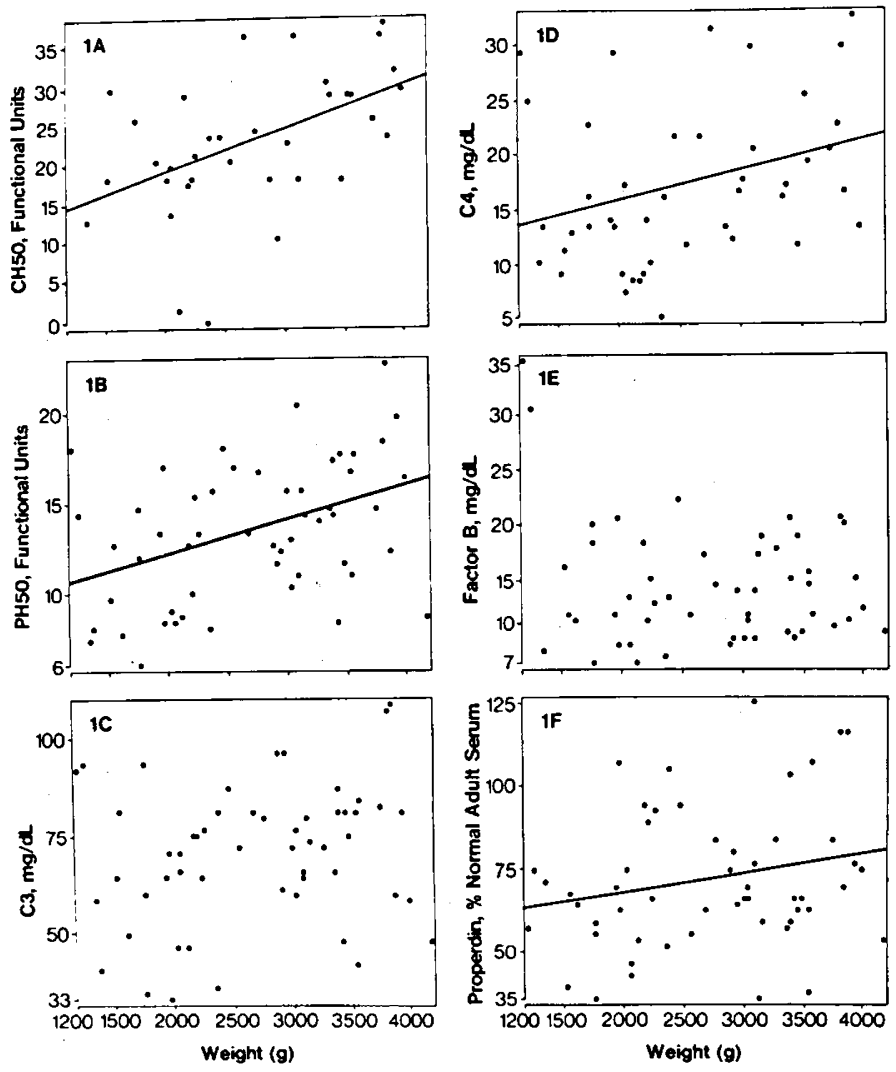

Fig. 1. Scatter plots showing the relationship between various components of the classical and alternative complement systems and birthweight. All but factor B and C3 were significantly correlated with weight, although there is much residual scatter.

way activities and complement components with weight are shown in Table 2 and Figure 1. The correlations of factor B and C3 with weight did not reach significance. The others attained at least the 0.05 level of significance, but nevertheless showed much residual scatter and only moderate correlations at best.

The correlations between the various complement levels are shown in Table 3. Twelve correlations were significant at the 0.01 level or beyond, two others were significant at the 0.05 level, and only the correlation between properdin and factor B was not significant.

\section{DISCUSSION}

Several other studies of the classical complement pathway in newborn sera have demonstrated that levels of whole complement activity and individual components are lower in cord sera than the levels in paired maternal sera or in normal adult sera $(1,3,6$, 13). As in these other studies, our data indicate that levels of both whole classical complement activity and the components C3 and $\mathrm{C} 4$ were grossly different than the levels in adult controls. Although the correlations between the $\mathrm{CH} 50$ and $\mathrm{C} 4$ levels and birth 
Table 3. Intercorrelations between complement level ${ }^{1}$

\begin{tabular}{lccccc}
\hline & \multicolumn{5}{c}{ Correlation coefficient } \\
\cline { 2 - 6 } & PH50 & C3 & C4 & B & Properdin \\
\hline CH50 & 0.74 & 0.55 & 0.68 & 0.51 & 0.43 \\
PH50 & & 0.70 & 0.75 & 0.53 & 0.41 \\
C3 & & & 0.52 & 0.57 & $0.28^{2}$ \\
C4 & & & & 0.57 & $0.28^{2}$ \\
B & & & & & $0.11^{3}$ \\
\hline
\end{tabular}

' All correlations significant at least at the 0.01 level except when noted.

${ }^{2}$ Correlation significant at 0.05 level.

${ }^{3}$ Correlation not significant.

weight were significant, there was a large scatter for these components as well as for C3. These results also are similar to other studies where the levels of the complement components were positively, but usually not significantly, correlated with birth weight $(5,6,13)$.

Because collection of sufficient numbers of extremely low birthweight infants was difficult, six sera from infants who developed severe illness were initially included. In these six sera, levels of some of the complement components were lower than would have been predicted on the basis of birthweight alone. The small numbers of infants in this study preclude drawing any conclusions about the relationship between development of severe infant illness and low complement levels. However, no relationship was seen between cord blood complement levels and mild newborn illness or maternal illness.

The results for the alternative complement pathway resemble those for the classical complement pathway, with the levels of activity and the specific components significantly lower than the adult controls. Levels of PH50 and properdin correlated significantly with birthweight, whereas factor B did not. The results of factor B levels are similar to the results obtained by Stossel $e$ t al. (15) and Alper et al. (2) in full term infants, which were $16.4 \mathrm{mg} /$ $\mathrm{dl} \pm 4.9$ and $12.0 \pm 5.1$, respectively.

The PH50 assay used here utilizes the observation that lysis of RaRBC in serum preferentially chelated with EGTA is independent of classical pathway activation (10). Polhill et al. (11) demonstrated immunoconversion of $\mathrm{C} 3$ and factor $\mathrm{B}$ during $\mathrm{RaRBC}$ activation of serum, confirming that the alternative pathway was activated. The endpoint lysis assay used in this study is dependent on the complement attack mechanism, $\mathrm{C} 5$ through $\mathrm{C} 9$, as well as the alternative activation sequence. Because the CH50 is also dependent on $\mathrm{C} 5$ through $\mathrm{C} 9$, a ratio of $\mathrm{CH} 50 / \mathrm{PH} 50$ would be an indication of the relative activities of the two activation sequences. For example, a $\mathrm{C} 2$ deficient serum which has normal alternative, but deficient classical, activation with a normal C5 through C9 attack mechanism has a $\mathrm{CH} 50$ of 0 , a normal PH50 and a CH50 to PH50 ratio of 0 (4). The ratio of CH50 to PH50 in the newborn sera was similar to the ratio in adult sera $(1.62 \pm 0.51$ and $1.85 \pm$ 0.32 , respectively, $P>0.1$ ), indicating that in newborn sera the alternative pathway activation sequence is deficient to the same degree as the classical activation sequence. The high degree of correlation between the PH50 and CH50 in the cord sera also supports this conclusion.

\section{REFERENCES AND NOTES}

1. Adinolfi, M. and Beck, S. E.: Human complement $\mathrm{C} 7$ and $\mathrm{C} 9$ in fetal and newborn sera. Arch. Dis. Child., 50: 562 (1975).

2. Alper, C. A., Boenisch, T., Watson, L.: Genetic polymorphism in human glycinerich beta-glycoprotein. J. Exp. Med., 135: 68 (1972).

3. Ballow, M., Fang, F., Good, R. A., Day, N. K.: Developmental aspects of complement components in the newborn: the presence of complement components and $\mathrm{C} 3$ proactivator (properdin factor B) in human colostrum. Clin. Exp. Immunol., 18: 257 (1974).

4. Colten, H. R.: (Personal communication)

5. Feinstein, P. A. and Kaplan, R.: The alternative pathway of complement activation in the neonate. Pediat. Res., 9: 803 (1975).

6. Fireman, P., Zuchowski, D. A., Taylor, P. M.: Development of human complement system. J. Immunol., 103: 25 (1969)

7. Johnston, R. B., Jr. and Stroud, R. M.: Complement and host defense against infection. J. Pediatr. 90: 169 (1977).

8. Mancini, G., Carbonara, A. O., Heremans, J. F. Immunochemical quantitation of antigens by single radial immunodiffusion. Immunochem., 2: 235 (1965).

9. Mayer, M. M.: Complement and complement fixation. In: E. A. Kabat and M. M. Mayer: Experimental Immunochemistry. p. 133 (Charles C Thomas, Co., Springfield, 1961).

10. Platts-Mills, T. A. E. and Ishizaka, K.: Activation of the alternate pathway of human complement by rabbit cells. J. Immunol., 113: 348 (1974).

11. Pohill, R. B., Jr., Pruitt, K. M., Johnston, R. B., Jr.: Assessment of alternative complement pathway activity in a continuously monitored hemolytic system. Fed. Proc., 34: 982 (1975).

12. Rapp, H. J. and Borsos, T.: The Molecular Basis of Complement Action. (Appleton-Century-Crofts, New York, 1970).

13. Sawyer, M. K., Forman, M. L., Kuplic, L. S., Stiehm, E. R.: Developmental aspects of the human complement system. Biol. Neonate, 19: 148 (1971).

14. Stiehm, E.: Fetal defense mechanisms. Am. J. Dis. Child. J29: 438 (1975).

15. Stossel, T. P., Alper, C. A., and Rosen, F. S.: Opsonic activity in the newborn: role of properdin. Pediatrics, 52: 134 (1973).

16. The authors thank Eileen McLanahan, Barbara Hauer, Susan Austin, and Cathleen Tapp for expert technical assistance.

17. This research was supported in part, by grants HD-09588 and AI-12970 from the National Institutes of Health and by a General Research Support Grant from the University of Arizona.

18. Requests for reprints should be addressed to: Robert C. Strunk, Department of Pediatrics, National Jewish Hospital \& Research Center, 3800 East Colfax, Denver, CO 80206, USA.

19. Received for publication February 16, 1978.

20. Accepted for publication June 8, 1978 . 